\title{
Manipulating magnetoelectric energy landscape in multiferroics
}

Yen-Lin Huang, ${ }^{1,2}$, Dmitri Nikonov (10 ${ }^{3}$, Christopher Addiego (1) ${ }^{4}$, Rajesh V. Chopdekar (10 ${ }^{5}$, Bhagwati Prasad', Lei Zhang (1) ${ }^{1}$, Jyotirmoy Chatterjee (1) ${ }^{6}$, Heng-Jui Liu (10 ${ }^{7}$, Alan Farhan (10 ${ }^{8}$, Ying-Hao Chu ${ }^{9}$, Mengmeng Yang ${ }^{10}$,

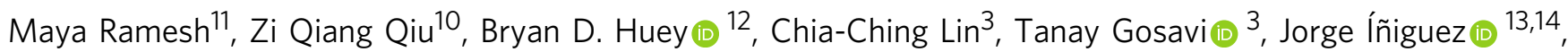

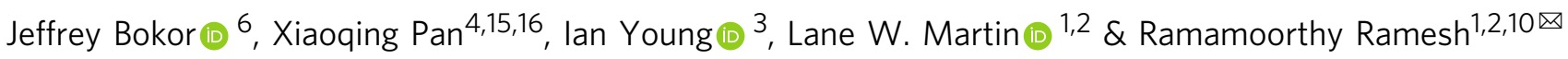

Magnetoelectric coupling at room temperature in multiferroic materials, such as $\mathrm{BiFeO}_{3}$, is one of the leading candidates to develop low-power spintronics and emerging memory technologies. Although extensive research activity has been devoted recently to exploring the physical properties, especially focusing on ferroelectricity and antiferromagnetism in chemically modified $\mathrm{BiFeO}_{3}$, a concrete understanding of the magnetoelectric coupling is yet to be fulfilled. We have discovered that La substitutions at the Bi-site lead to a progressive increase in the degeneracy of the potential energy landscape of the $\mathrm{BiFeO}_{3}$ system exemplified by a rotation of the polar axis away from the $\langle 111\rangle_{\mathrm{pc}}$ towards the $\langle 112\rangle_{\mathrm{pc}}$ discretion. This is accompanied by corresponding rotation of the antiferromagnetic axis as well, thus maintaining the right-handed vectorial relationship between ferroelectric polarization, antiferromagnetic vector and the Dzyaloshinskii-Moriya vector. As a consequence, $\mathrm{La}^{-\mathrm{BiFeO}_{3}}$ films exhibit a magnetoelectric coupling that is distinctly different from the undoped $\mathrm{BiFeO}_{3}$ films.

\footnotetext{
${ }^{1}$ Department of Materials Science and Engineering, University of California, Berkeley, Berkeley, CA 94720, USA. ${ }^{2}$ Materials Sciences Division, Lawrence Berkeley Laboratory, Berkeley, CA 94720, USA. ${ }^{3}$ Exploratory Integrated Circuits, Components Research, Intel Corp., Hillsboro, Oregon 97124, USA. ${ }^{4}$ Department of Physics and Astronomy, University of California, Irvine, Irvine, CA 92697, USA. ${ }^{5}$ Advanced Light Source, Lawrence Berkeley National Laboratory, Berkeley, CA 94720, USA. ${ }^{6}$ Electrical Engineering and Computer Sciences, University of California, Berkeley, Berkeley, CA 94720, USA. ${ }^{7}$ Department of Materials Science and Engineering, National Chung Hsing University, Taichung 402, Taiwan. ${ }^{8}$ Department of Applied Physics, Aalto University School of Science, Espoo Fl-00076, Finland. ${ }^{9}$ Department of Materials Science and Engineering, National Chaio Tung University, Hsinchu 300, Taiwan. ${ }^{10}$ Department of Physics, University of California, Berkeley, Berkeley, CA 94720, USA. ${ }^{11}$ Department of Physics, University of California, Irvine, Santa Cruz, CA 95064, USA. ${ }^{12}$ Department of Materials Science and Engineering, University of Connecticut, Storrs, CT 06269, USA. ${ }^{13}$ Materials Research and Technology Department, Luxembourg Institute of Science and Technology (LIST), 5 avenue des Hauts-Fourneaux, L-4362 Esch/Alzette, Luxemburg.

${ }^{14}$ Physics and Materials Science Research Unit, University of Luxembourg, 41, rue du Brill, L-4422 Belvaux, Luxembourg. ${ }^{15}$ Department of Materials Science and Engineering, University of California, Irvine, Irvine, CA 92697, USA. ${ }^{16}$ Irvine Materials Research Institute, University of California, Irvine, Irvine, CA 92697 , USA. email: rramesh@berkeley.edu
} 
M agnetoelectric multiferroics are materials which possess two or more order parameters simultaneously and, more importantly, exhibit coupling between the spin and charge degrees of freedom ${ }^{1,2}$. Bismuth ferrite $\left(\mathrm{BiFeO}_{3}\right)$ is by far the most studied and characterized multiferroic in part because it exhibits robust order parameters-ferroelectricity (P), antiferromagnetism $(\mathbf{L})$, a weak ferromagnetic moment $\left(\mathbf{M}_{\mathbf{C}}\right)$ induced by canting of the antiferromagnetically aligned spins, and magnetoelectric coupling between these order parameters $(\mathbf{P}, \mathbf{L}$, $\mathbf{M}_{\mathbf{C}}$ ) well above room temperature ${ }^{3}$. Because of the nature of its magnetoelectric coupling, $\mathrm{BiFeO}_{3}$ holds significant promise to trigger the development of low-power consumption memory and logic devices ${ }^{4-6}$. The strong spontaneous polarization in $\mathrm{BiFeO}_{3}$, however, results in a correspondingly large coercive voltage [described by the classic Landau double-well (Fig. 1a)], which requires adjustment to enable low-voltage applications ${ }^{7,8}$. Bulk $\mathrm{BiFeO}_{3}$ has a ferroelectric polarization of $\sim 90 \mu \mathrm{C} \mathrm{cm}^{-2}$ pointing along the $\langle 111\rangle_{\mathrm{pc}}$ (where pc refers to the pseudo-cubic notation) with a rhombohedrally distorted crystal structure (space group $R 3 c)^{9}$. Unlike most conventional displacive ferroelectrics (e.g., $\mathrm{BaTiO}_{3}$ and $\mathrm{PbTiO}_{3}$ ) which have polarization induced by the hybridization between the empty transition metal $d$ orbital and the filled oxygen $2 p$-orbital, the polarization in $\mathrm{BiFeO}_{3}$ primarily originates from the stereo-chemically active lone pair in the form of the $A$-site $\mathrm{Bi}^{3+} 6 s^{2}$ electrons ${ }^{10,11}$. This has motivated - because of the intimate connection with the polarization - extensive studies of chemical substitution of the $A$ site of $\mathrm{BiFeO}_{3}$ including using rare-earth elements such as $\mathrm{La}^{3+}, \mathrm{Sm}^{3+}$, and $\mathrm{Dy}^{3+}$ because of their similar ionic radius and isovalent chemistry to bismuth. A systematic change in ferroelectric ordering is indeed induced by rareearth substitution for bismuth, including a reduction of the Curie temperature, formation of an antiferroelectric phase, etc ${ }^{12-16}$. On the other hand, isovalent chemical substitution of the $A$ site is not expected to alter the antiferromagnetism in $\mathrm{BiFeO}_{3}$ and, in fact, only minimal effects on the Néel temperature are reported ${ }^{17}$. More importantly, however, is the impact of such rare-earth substitution for the evolution of the coupling mechanism between the magnetic and ferroelectric order. Despite having profound implications for material and device function, few studies have considered this. In turn, in the pursuit of the important technological question of how to enable low-voltage control of magnetism, foundational studies to unveil the correlation between $\mathbf{P}, \mathbf{L}, \mathbf{M}_{\mathbf{C}}$, and their switching pathways in, for example, rare-earth lanthanum-substituted $\mathrm{BiFeO}_{3}\left(\mathrm{Bi}_{1-x} \mathrm{La}_{x} \mathrm{FeO}_{3}\right)$ thin films are a key step towards addressing the material requirements for low-power spintronics.

\section{Results}

Polarization reduction and rotation in $\mathrm{Bi}_{1-x} \mathrm{La}_{x} \mathrm{FeO}_{3}$. To study the evolution of the order parameters, $\mathbf{P}, \mathbf{L}$, and $\mathbf{M}_{\mathbf{C}}$, under chemical pressure, we explore a model system consisting of $(001)_{\mathrm{pc}}$ oriented $\mathrm{Bi}_{1-x} \mathrm{La}_{x} \mathrm{FeO}_{3}(x=0,0.1,0.15,0.2$, and 0.3$)$ thin films grown on a conducting layer of $\mathrm{SrRuO}_{3}$ on insulating $\mathrm{DyScO}_{3}$ $(110)_{O}$ single crystal substrates (where $\mathrm{O}$ refers to orthorhombic indices), all grown by pulsed-laser deposition (Methods). Rhombohedral $\mathrm{BiFeO}_{3}$ can be thought of as exhibiting a pseudocubic perovskite unit cell which has been distorted along the $\langle 111\rangle_{\mathrm{pc}}$ body diagonal resulting in a spontaneous polarization along that axis. On the other hand, in $\mathrm{Bi}_{1-x} \mathrm{La}_{x} \mathrm{FeO}_{3}$, increasing amounts of lanthanum drives a suppression of the magnitude of and, possibly reorient, the polarization direction (Fig. 1b). As a consequence, chemical substitution could also provide a methodology to reduce the free-energy barrier for ferroelectric switching described by a classical Landau model (Fig. 1a) and experimentally demonstrated in polarization hysteresis loops (Fig. 1c). The polarization hysteresis loops, as a function of lanthanum content $\left(\mathrm{Bi}_{1-x} \mathrm{La}_{x} \mathrm{FeO}_{3}\right.$ with $x=0,0.1,0.15,0.20$, and 0.3 ), reveal a systematic suppression of remanent polarization (from $65 \mu \mathrm{C} \mathrm{cm}^{-2}$ for $x=0$ to $18 \mu \mathrm{C} \mathrm{cm}^{-2}$ for $x=0.2$ ) and a corresponding reduction of the average coercive field (from $158 \mathrm{kV} \mathrm{cm}^{-1}$ for $x=0$ to $106 \mathrm{kV} \mathrm{cm}^{-1}$ for $x=0.2$ ).

Reciprocal space maps (RSMs) about the $203_{\mathrm{pc}}$-diffraction condition of the films confirm the change in crystal structure (Supplementary Figs. 1, 2) and were used to extract the rhombohedral angles as well as the in-plane and out-of-plane lattice constants for this series of samples. We observe that the rhombohedral distortion decreases with lanthanum substitution while maintaining the imposed strain from the $\mathrm{DyScO}_{3}$ substrate (i.e., all films are coherently strained to the substrate such that the in-plane lattice constant of the film remains the same despite changing the chemistry). This indicates that a phase
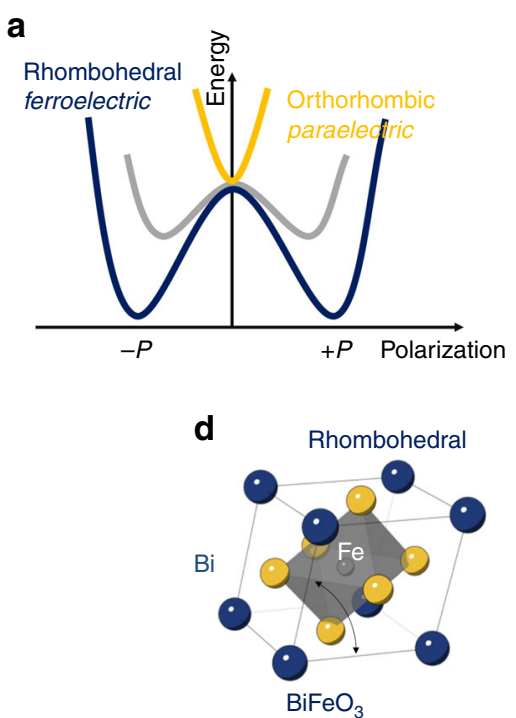
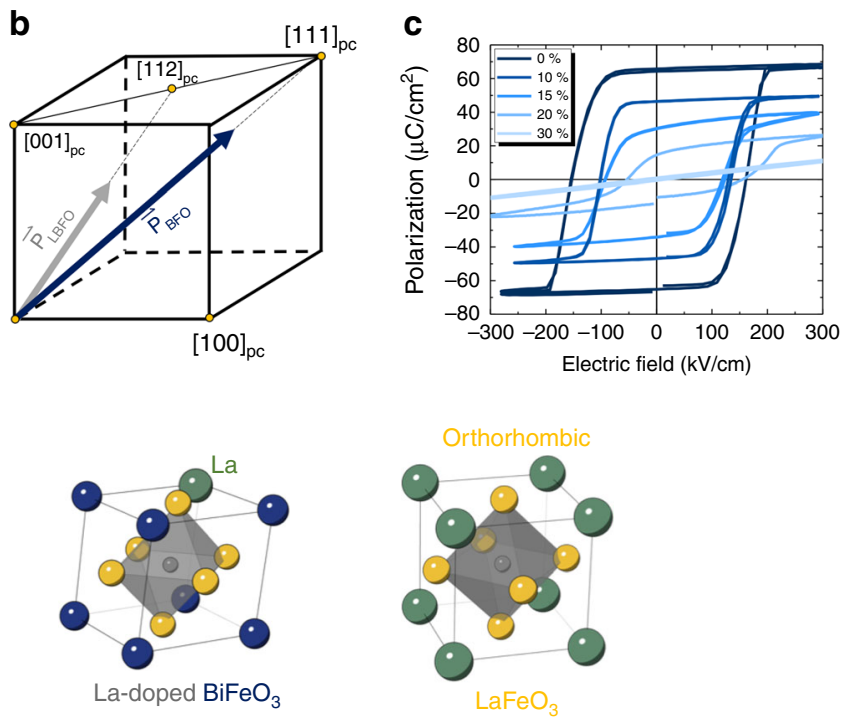

Fig. 1 Ferroelectric ordering in $\mathbf{B i}_{\mathbf{1}_{-\mathbf{x}}} \mathbf{L} \mathbf{a}_{\mathbf{x}} \mathbf{F} \mathbf{F O}_{\mathbf{3}}$. a Schematic for the energy landscape of the phase transition induced by lanthanum substitution in BiFeO described by Landau theory. b Schematic for the ferroelectric polarization rotation (from $\mathrm{BiFeO}_{3}$ : $[111]_{\mathrm{pc}}$ to $\mathrm{Bi}_{0.85} \mathrm{La}_{0.15} \mathrm{FeO}_{3}$ : $[112]_{\mathrm{pc}}$ ) and suppression of ferroelectric polarization induced by lanthanum substitution in $\mathrm{BiFeO}_{3}$. c $P$ - $E$ measurements for different substitution levels of lanthanum in $100-\mathrm{nm}$-thick $\mathrm{BiFeO}_{3}$ films. d The schematics illustrate the evolution of crystal symmetry of bismuth ferrite (rhombohedral) to lanthanum ferrite (orthorhombic). 


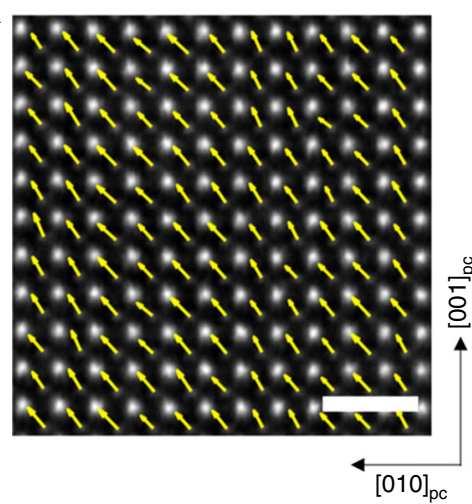

b

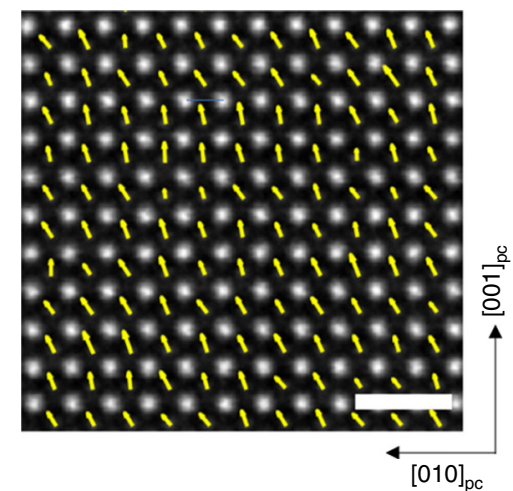

C

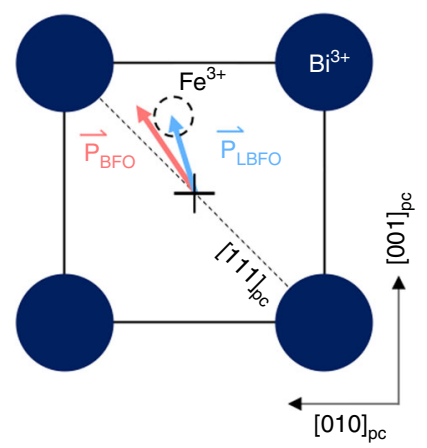

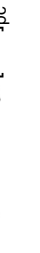

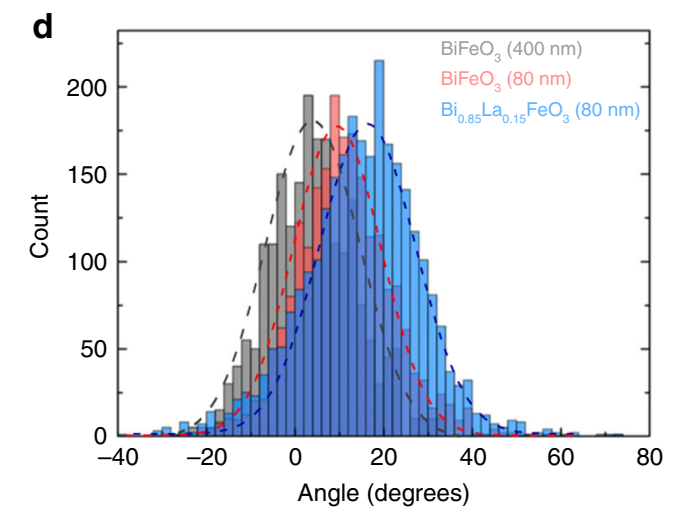

Fig. 2 Atomic images, polarization mapping, and change of polarization in $\mathbf{B i F e O}_{\mathbf{3}}$ and $\mathbf{B i}_{\mathbf{0 . 8 5}} \mathbf{L a}_{\mathbf{0} .15} \mathbf{F e O}_{\mathbf{3}}$ thin films. a, $\mathbf{b}$ show the $\mathrm{HAADF}-\mathrm{STEM}$ images of $\mathrm{BiFeO}_{3}$ and $\mathrm{Bi}_{0.85} \mathrm{La}_{0.15} \mathrm{FeO}_{3}$, respectively, with the polarization mapping of the Fe atoms overlaid. The scale bar is $1 \mathrm{~nm}$. c Schematic of ferroelectric polarization in $\mathrm{BiFeO}_{3} / \mathrm{Bi}_{0.85} \mathrm{La}_{0.15} \mathrm{FeO}_{3}$ unit cell. The vectors in $(\mathbf{a}, \mathbf{b})$ were extracted from the displacement of $\mathrm{Fe}^{3+}$ position to the mass center of four $\mathrm{Bi}^{3+}$. d Histogram of polar distribution shows that the ferroelectric polarizations rotate $3.6^{\circ}, 10.9^{\circ}$ and $16.1^{\circ}$ away from $[111]_{\mathrm{pc}}$ in $400-\mathrm{nm}-\mathrm{thick} \mathrm{BiFeO} 3$ (gray), 80-nm-thick $\mathrm{BiFeO}_{3}$ (red) and 80 -nm-thick $\mathrm{Bi}_{0.85} \mathrm{La}_{0.15} \mathrm{FeO}_{3}$ (blue), respectively.

transition from rhombohedral to orthorhombic can be driven by such a chemical substitution ${ }^{13}$.

While it is clear that lanthanum substitution can reduce the magnitude of the measured polarization and the coercive field, the specific nature of this reduction (i.e., does it arise from polarization rotation, reduction of polarization magnitude, or some combination therein) remains to be determined. To directly visualize the nature of the change in polarization at the microscopic scale, we carried out high-angle annular dark field scanning transmission electron microscopy (HAADF-STEM, "Methods" section) imaging on both the $\mathrm{B}_{0.85} \mathrm{La}_{0.15} \mathrm{FeO}_{3}$ and $\mathrm{BiFeO}_{3}$ thin films. From this, it is possible to extract quantitative local information about the direction of the polarization vector by measuring the displacement of the B-site cation (i.e., iron) to the mass center of the four cations at the unit-cell corners (i.e., bismuth and lanthanum) across each unit cell in the image (Fig. 2c). For $\mathrm{BiFeO}_{3}$ (Fig. 2a), the extracted map of polarization (which is a projection upon a $(100)_{\mathrm{pc}}$ ) reveals a strong tendency for the polarization to point along a diagonal direction consistent with polarization pointing along a $[111]_{\mathrm{pc}}$ - but slightly tilted towards $[001]_{p c}$ (as shown in Fig. 2d). (this is intuitively expected in the $0.4 \%$ compressively strained $\mathrm{BiFeO}_{3}$, which should drive a rotation of the polarization towards the out-ofplane direction $\sim 10.9^{\circ}$ from [111] $]_{\mathrm{pc}}$ as shown in Fig. $\left.2 \mathrm{~d}\right)^{18}$ In contrast, for the $\mathrm{Bi}_{0.85} \mathrm{La}_{0.15} \mathrm{FeO}_{3}$ (Fig. 2b), the extracted map of polarization (which again is a projection on a $(100)_{\mathrm{pc}}$ ) reveals a clear rotation of the polarization away from a $[111]_{\mathrm{pc}}$ towards a $[112]_{\mathrm{pc}}$ by $\sim 16.1^{\circ}$ (the clockwise rotation is defined as positive direction), revealed by the histograms of measured polar vector directions across the entire area analyzed for both the $\mathrm{BiFeO}_{3}$ and
$\mathrm{Bi}_{0.85} \mathrm{La}_{0.15} \mathrm{FeO}_{3}$ (Fig. 2d). We also measured the polarization rotation of the $400-\mathrm{nm}$-thick BFO sample where the polarization only deviates $\sim 3.6^{\circ}$ from $[111]_{\mathrm{pc}}$ (Fig. $2 \mathrm{~d}$ ). For reference, the angle between the $[111]_{\mathrm{pc}}$ and $[112]_{\mathrm{pc}}$ in this projection is $19.4^{\circ}$.

This, in conjunction with the experimentally measured remanent polarization in $\mathrm{BiFeO}_{3}$ and $\mathrm{Bi}_{0.85} \mathrm{La}_{0.15} \mathrm{FeO}_{3}$ projected along $[001]_{p c}$ (Fig. 1c), provides us with an estimate of the ferroelectric polarization for such a structurally distorted and chemically substituted, $\mathrm{Bi}_{0.85} \mathrm{La}_{0.15} \mathrm{FeO}_{3}$ film (the details are captured in the Supplementary Information Fig. 1). We calculate the ferroelectric polarization in $\mathrm{Bi}_{0.85} \mathrm{La}_{0.15} \mathrm{FeO}_{3}$ to be $\sim 44 \mu \mathrm{Ccm}{ }^{-2}$, i.e., a significant reduction from the spontaneous polarization of $90 \mu \mathrm{C} \mathrm{cm}^{-2}$ in pure $\mathrm{BiFeO}_{3}$. Thus, we conclude that incorporation of $15 \%$ lanthanum into the parent $\mathrm{BiFeO}_{3}$ structure alters the nature of the polarization (i.e., both the magnitude and the direction of the polar vector), consistent with prior work ${ }^{14-16,19}$.

Armed with an understanding from X-ray diffraction and STEM analyses on how the material structure is evolving, we turned to piezoresponse force microscopy (PFM, "Methods" section) for an analysis of the evolution of the mesoscale domain structure, which similarly reveals marked differences. The as-grown domain structure of 20-nm-thick $\mathrm{BiFeO}_{3}$ features well-ordered stripe domain patterns (Fig. 3a), while that of 20-nm-thick $\mathrm{Bi}_{0.85} \mathrm{La}_{0.15} \mathrm{FeO}_{3}$ has no stripes, but exhibits a complex domain pattern (Fig. 3c). This is a consequence of weakened rhombohedral distortion (and a concomitant reduction in structural anisotropy), which is consistent with both the X-ray diffraction and STEM analyses above. In turn, one would expect that these crystal and domain structure differences will likely lead to corresponding differences in the mesoscale ferroelectric switching behavior - which can be probed via a combination of both 

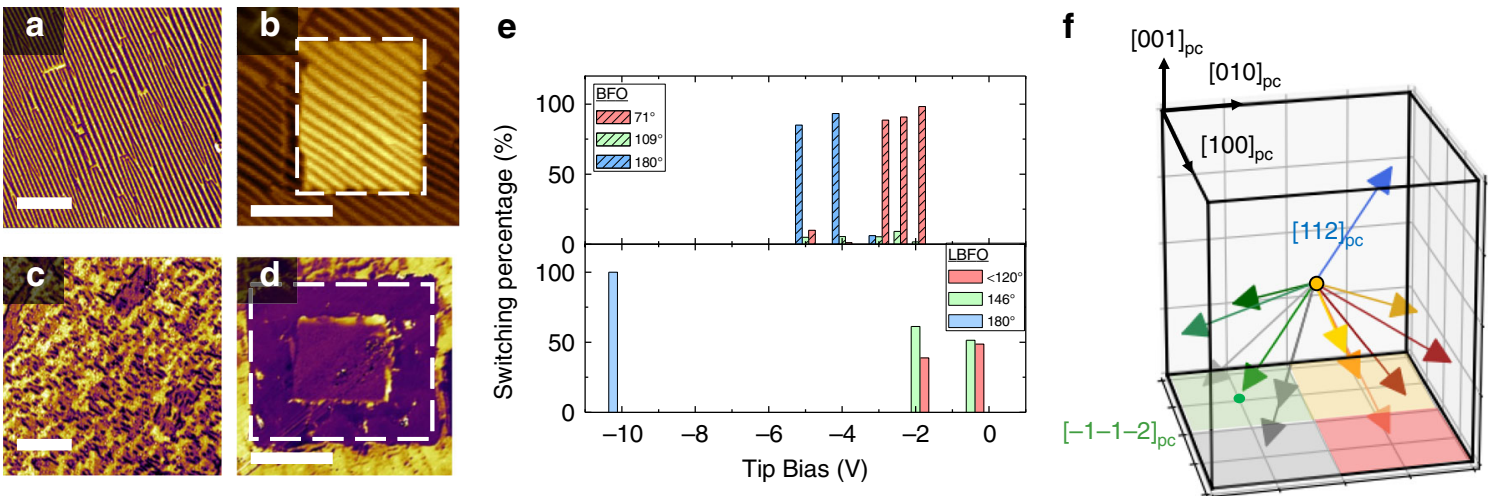

Fig. 3 Ferroelectric switching in $\mathbf{B i F e O}_{\mathbf{3}}$ and $\mathbf{B i}_{\mathbf{0 . 8 5}} \mathbf{L a}_{\mathbf{0 . 1 5}} \mathbf{F e O}_{\mathbf{3}}$ revealed by $\mathbf{P F M}$. $\mathbf{a}$, $\mathbf{c}$ The as-grown IP-PFM images show different domain patterns in 20-nm-thick $\mathrm{BiFeO}_{3}$ and $\mathrm{Bi}_{0.85} \mathrm{La}_{0.15} \mathrm{FeO}_{3}$ thin films, respectively. $\mathbf{b}$ In-plane PFM images of 20-nm-thick $\mathrm{BiFeO}_{3}$ after PFM electric poling with - $5 \mathrm{~V}$ (upward polarized). $\mathbf{d}$ In-plane PFM images of 20 -nm-thick $\mathrm{Bi}_{0.85} \mathrm{La}_{0.15} \mathrm{FeO}_{3}$ after PFM electric poling with $-10 \mathrm{~V}$ (upward polarized). The scale bar is $1 \mu \mathrm{m}$. e The summary of the polarization switching angles for 20-nm-thick $\mathrm{BiFeO}_{3}$ and $\mathrm{Bi}_{0.85} \mathrm{La}_{0.15} \mathrm{FeO}_{3}$ thin films. $\mathbf{f}$ Schematic of ferroelectric polarization switching in $\mathrm{Bi}_{0.85} \mathrm{La}_{0.15} \mathrm{FeO}_{3}$ thin film.

lateral and vertical PFM ("Methods" section). For a 20-nm-thick $\mathrm{BiFeO}_{3}$ heterostructure (Fig. 3b), for example, application of a $-5 \mathrm{~V}$ DC voltage to the PFM tip reveals, as expected, classical $180^{\circ}$ switching of polarization (i.e., reversed phase contrast in both the lateral and vertical PFM channels; Supplementary Information and Supplementary Figs. 3-6). For the same thickness $\mathrm{Bi}_{0.85} \mathrm{La}_{0.15} \mathrm{FeO}_{3}$ heterostructure (Fig. 3d), however, $180^{\circ}$ switching of the polarization cannot be achieved unless a voltage very close to the breakdown voltage $\left(\sim 10 \mathrm{~V}, 5 \mathrm{MV} \mathrm{cm}^{-1}\right)$ is applied. We capture the statistics of this difference in switching behavior in Fig. 3e, which shows histograms of the switching process as a function of voltage for both 20-nm-thick $\mathrm{BiFeO}_{3}$ and $\mathrm{Bi}_{0.85} \mathrm{La}_{0.15} \mathrm{FeO}_{3}$, from which a summary of the differences in switching behavior can be constructed (Fig. 3f). Unlike $\mathrm{BiFeO}_{3}, \mathrm{Bi}_{0.85} \mathrm{La}_{0.15} \mathrm{FeO}_{3}$ has the polarization vector pointing along $\sim\langle 112\rangle_{\mathrm{pc}}$ resulting in a larger number of possible switching paths (e.g., for the $\langle 112\rangle_{\mathrm{pc}}$, there are 12 possible switching directions (illustrated in Fig. 3f), of which only one corresponds to a full $180^{\circ}$ switch; in comparison, there are only 4 possible switching directions for the $\langle 111\rangle_{\mathrm{pc}}$ polarization direction in $\mathrm{BiFeO}_{3}$. The details are laid out in the Supplementary Information and Supplementary Fig. 4a. This high energy penalty of $180^{\circ}$ switching in $\mathrm{Bi}_{0.85} \mathrm{La}_{0.15} \mathrm{FeO}_{3}$ could be associated with the energy consumption to accommodate ferroelastic deformation during multiple-step switching similar to that observed in $\mathrm{BiFeO}_{3}{ }^{20}$.

With this understanding of the nature of the ferroelectric polarization and its switching in $\mathrm{Bi}_{0.85} \mathrm{La}_{0.15} \mathrm{FeO}_{3}$, we now turn our attention to exploring the magnetic order, namely, $\mathbf{L}$ and $\mathbf{M}_{\mathbf{C}}$. The parent phase $\mathrm{BiFeO}_{3}$ has a $G$-type antiferromagnetic structure, wherein the iron magnetic moments are ferromagnetically coupled within $\{111\}_{\mathrm{pc}}$ and antiferromagnetically aligned between adjacent $\{111\}_{\mathrm{pc}}$. Further, the anti-symmetric Dzyaloshinskii-Moriya exchange interaction (DMI), manifests from spin-orbit coupling and allows for the formation of a macroscopic magnetization $\left(\mathbf{M}_{\mathbf{C}}\right)$ caused by a canting of the antiferromagnetic sublattices $^{21}$. The DMI has a simple energy expression:

$$
E=-D \times\left(L \times M_{\mathrm{C}}\right),
$$

where $\mathbf{D}$ is the DMI vector, $\mathbf{L}$ is the antiferromagnetic vector, and $\mathbf{M}_{\mathbf{C}}$ is the canted magnetic moment vector ${ }^{22}$. In bulk $\mathrm{BiFeO}_{3}$, to minimize the free energy, the neighboring magnetic sublattices $\left(\mathbf{M}_{1}, \mathbf{M}_{\mathbf{2}}\right)$, which define the antiferromagnetic axis: $\mathbf{L}=\mathbf{M}_{\mathbf{1}}-\mathbf{M}_{\mathbf{2}}$, should be canted leading to a $\mathbf{M}_{\mathbf{C}}$ that is perpendicular to $\mathbf{L}$ and rotates within the $\{111\}_{\mathrm{pc}}$ magnetic easy planes with a periodicity of $\sim 62 \mathrm{~nm}$ due to the non-degeneracy of magnetic anisotropy ${ }^{23}$. We also note that this spin-cycloid structure is known to be suppressed in high-quality strained epitaxial thin films in which density functional calculations suggest that the $\mathbf{L}$ should lie along $\langle 110\rangle_{\mathrm{pc}}$ or $\langle 112\rangle_{\mathrm{pc}}$ with the $\{111\}_{\mathrm{pc}}{ }^{22,24-26}$. Regardless of the existence of the spin-cycloid structure, these three vectors, $\mathbf{D}, \mathbf{L}$, and $\mathbf{M}_{\mathbf{C}}$ naturally form a right-handed multiferroic vector system (D aligns with $\langle 111\rangle_{\mathrm{pc}}$, which is parallel to the ferroelectric polarization $)^{22}$. This right-handed vector system is the crucial underpinning behind magnetoelectric coupling ${ }^{20}$. It has been shown that under large compressive strain (for example imposed by epitaxial strain from a $\mathrm{SrTiO}_{3}$ substrate $[-1.4 \%$ compressive $]$ ), the single ion anisotropy component of the Hamiltonian can begin to dominate ${ }^{27}$; however, given the significantly smaller strain imposed by the $\mathrm{DyScO}_{3}$ substrate in our case, we expect the magnetic structure to be still controlled by the DMI in the crystal. With this as the background, an understanding of how the magnetic order parameters develop in $\mathrm{Bi}_{0.85} \mathrm{La}_{0.15} \mathrm{FeO}_{3}$ as a consequence of the significant rotation of the polarization vector away from the $\langle 111\rangle_{\mathrm{pc}}$ direction is a critical and fundamentally important question. Specifically, we are interested in two questions: (1) do $\mathbf{P}, \mathbf{L}$, and $\mathbf{M}_{\mathbf{C}}$ still form a right-handed system in $\mathrm{Bi}_{0.85} \mathrm{La}_{0.15} \mathrm{FeO}_{3}$ ? and (2) what happens to $\mathbf{P}, \mathbf{L}$, and $\mathbf{M}_{\mathbf{C}}$ when the polarization is switched with an out-of-plane electric field?

Probing magnetic order and magnetoelectric coupling in $\mathbf{B i}_{\mathbf{1}}$ ${ }_{-x} \mathrm{La}_{x} \mathrm{FeO}_{3}$. To address these questions, we used X-ray magnetic linear dichroism-photoemission electron microscopy (XMLDPEEM) combined with PFM to establish the correlation between the order parameters in $\mathrm{Bi}_{1-x} \mathrm{La}_{x} \mathrm{FeO}_{3}$ thin films. The geometry of the XMLD-PEEM experiment is shown with two angular dependences ( $\boldsymbol{\alpha}$ for the linear polarization of the X-rays and $\boldsymbol{\Phi}$ for the azimuthal in-plane direction of the sample; Fig. $4 \mathrm{a}$ ). Here we used two types of samples, $\mathrm{BiFeO}_{3}$ and $\mathrm{Bi}_{0.85} \mathrm{La}_{0.15} \mathrm{FeO}_{3}$, with two different thicknesses, 20 and $80 \mathrm{~nm}$ (see sample preparation details in the Method section). Typical of high-quality $\mathrm{BiFeO}_{3}$ heterostructures, two-variant polarization, periodic stripe domains are observed for both $80 \mathrm{~nm}$ (Fig. 4b) and $20 \mathrm{~nm}$ (Fig. 4d) heterostructures ${ }^{28}$. We performed electrical switching of the ferroelectric domain structure (shown in the box) and, by careful control of the applied bias to the PFM tip (Methods and Supplementary Information), we are able to obtain stripy, twovariant domain structures after switching. Such a model system (both the 80 - and 20 -nm-thick $\mathrm{BiFeO}_{3}$ heterostructures) provides the reference frame to study the antiferromagnetic ordering $\mathbf{L}$. XMLD-PEEM is a powerful tool for the investigation of magnetic ordering for many ferromagnetic and antiferromagnetic systems 
with high spatial resolution and elemental specificity ${ }^{29-31}$. This approach allows one to image the antiferromagnetic domains (Fig. 4c, e) with chemical specificity (here the images were taken at the iron $L_{2}$ edge; Methods). This specific energy (peak) was chosen for imaging because both antiferromagnetic and ferroelectric ordering contribute strongly to the dichroism at the iron $L_{3}$ edge while the dichroism at the $L_{2}$ edge is dominated by magnetic order $25,32,33$. Consistent with previous studies, we
$\mathrm{Bi}_{0.85} \mathrm{La}_{0.15} \mathrm{FeO}_{3}$ samples, while in $\mathrm{BiFeO}_{3}$ samples, the canted moment stays in the plane $(110)_{\mathrm{pc}}$. This can be validated by exploring the coupling of the $\mathbf{M}_{\mathbf{C}}$ to an external magnet. For example, an in-plane magnetized $\mathrm{Co}_{0.9} \mathrm{Fe}_{0.1}$ layer shows a progressively lower exchange coupling field, as illustrated in Supplementary Fig. 12, supporting the notion that the canted moment is tilting away from the in-plane direction towards the out-of-plane, as lanthanum is added. In contrast, a ferromagnetic
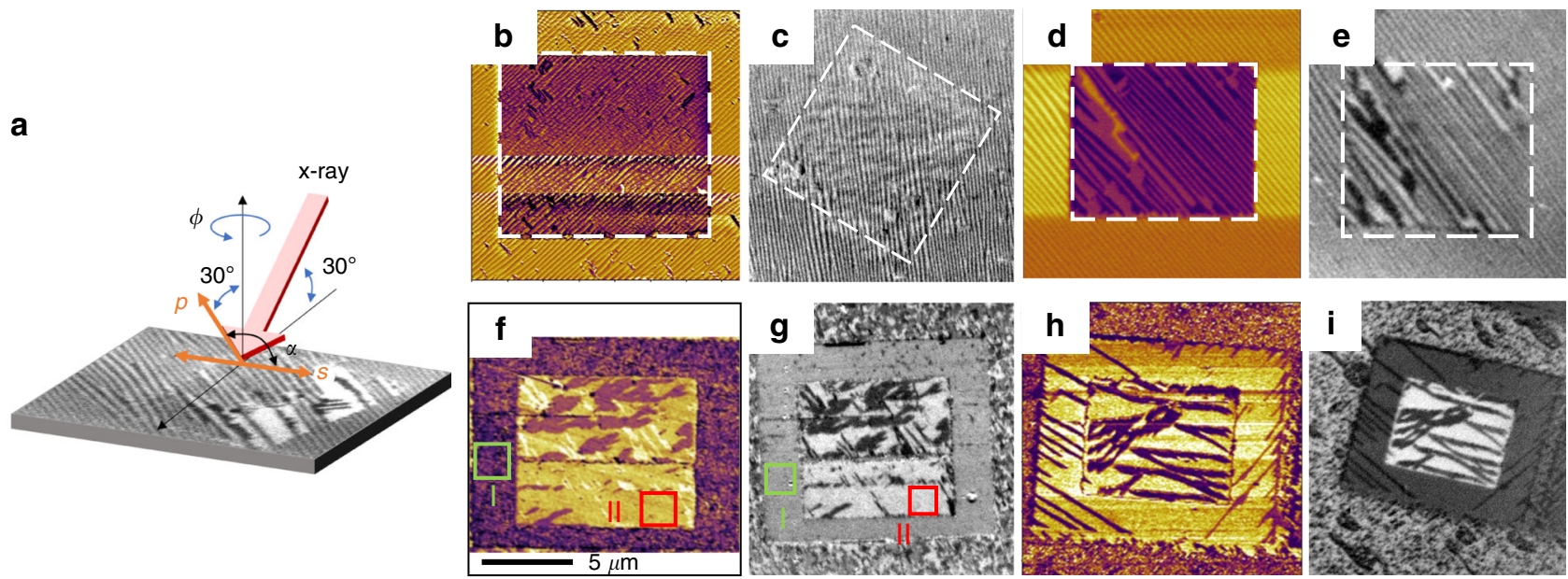

Fig. 4 PFM and XMLD-PEEM images of $\mathbf{B i F e O}_{\mathbf{3}}$ and $\mathbf{B i}_{\mathbf{0 . 8 5}} \mathbf{L a}_{\mathbf{0 . 1 5}} \mathbf{F e O}_{\mathbf{3}}$ thin films. a Schematic of the XMLD-PEEM experimental geometries used to probe the angle dependence $(\boldsymbol{\Phi})$, linear dichroism. Linear polarizations: $\boldsymbol{\alpha}=0^{\circ}$; Linear polarization p: $\boldsymbol{\alpha}=90^{\circ}$. $\mathbf{b}, \mathbf{c}$ In-plane-PFM and XMLD-PEEM images of 80-nm-thick $\mathrm{BiFeO}_{3}$. d, e In-plane-PFM and XMLD-PEEM images of 20-nm-thick BiFeO 3 . f, $\mathbf{g}$ In-plane-PFM and XMLD-PEEM images of 80-nm-thick $\mathrm{Bi}_{0.85} \mathrm{La}_{0.15} \mathrm{FeO}_{3}$. The green/red boxes represent the positive/negative polarized domain I/II. h, i In-plane-PFM and XMLD-PEEM images of 20-nm-thick $\mathrm{Bi}_{0.85} \mathrm{La}_{0.15} \mathrm{FeO}_{3}$.

observe a one-to-one correlation of ferroelectric and antiferromagnetic domains in both the 80- and 20-nm-thick $\mathrm{BiFeO}_{3}$ films due to the inherent coupling of polarization and magnetization in this material. By extracting the intensity of different $\boldsymbol{\alpha}$ and $\boldsymbol{\Phi}$ XMLD-PEEM images in different domains (see the analysis of $\mathbf{L}$ in Supplementary Information and Supplementary Figs. 7, 9), we find that the $\mathbf{L}$ in both the 80 - and 20 -nm-thick $\mathrm{BiFeO}_{3}$ heterostructures is approximately parallel to a $\langle 112\rangle_{\mathrm{pc}}$ before and after electrical switching.

Similar analysis was completed on the $\mathrm{Bi}_{0.85} \mathrm{La}_{0.15} \mathrm{FeO}_{3}$ heterostructures, which also show electrically switched antiferromagnetic domains with one-to-one correlation to the ferroelectric domains (Fig. $4 \mathrm{f}-\mathrm{i}$ ). Fitting of the XMLD intensity versus $\boldsymbol{\alpha}$ and $\boldsymbol{\Phi}$ in the antiferromagnetic domains for $\mathrm{Bi}_{0.85} \mathrm{La}_{0.15} \mathrm{FeO}_{3}$ (Supplementary Figs. 8-10), however, show that the $\mathbf{L}$ in domain $\mathrm{I}$ is along $\sim[641]_{\mathrm{pc}}$ and the $\mathbf{L}$ in domain II is along $\sim[10-2]_{\mathrm{pc}}$, which means the $\mathbf{L}$ rotates by $\sim 105^{\circ}$, consistent with the $\mathbf{P}$ switching (Fig. 3f). This also points to the fact that the easy magnetic plane for $\mathrm{Bi}_{0.85} \mathrm{La}_{0.15} \mathrm{FeO}_{3}$ is no longer in the $\{111\}_{\mathrm{pc}}$ but most likely in the $\{121\}_{\mathrm{pc}}$ (Supplementary Fig. 11). We attribute this change in the magnetic easy plane to the consequence of the rotation of the ferroelectric polarization in the $\mathrm{Bi}_{0.85} \mathrm{La}_{0.15} \mathrm{FeO}_{3}$ (Fig. 1). That is, as a consequence of the polarization in $\mathrm{Bi}_{0.85} \mathrm{La}_{0.15} \mathrm{FeO}_{3}$ pointing (approximately) along the $[112]_{\mathrm{pc}}, \mathbf{L}$ and $\mathbf{M}_{\mathbf{C}}$ are expected to follow the motion of the polarization accordingly (i.e., polarization is switched from, for example, $[1-12]_{\mathrm{pc}}$ to $\left.[21-1]_{\mathrm{pc}}\right)$.

Based on the ferroelectric data in Fig. 3 and the antiferromagnetic data in Fig. 4, one can calculate the canted moment direction, $\mathbf{M}_{\mathbf{C}}$, using eq. (1). Our calculations indicate that the canted moment direction switched from $\mathbf{M}_{\mathbf{C I}} \sim\left[\begin{array}{lll}-7 & 13 & 10\end{array}\right]_{\mathrm{pc}}$ to $\mathbf{M}_{\mathrm{CII}} \sim[251]_{\mathrm{pc}}$ with the applied electric field in the multilayer with perpendicular magnetic anisotropy, such as $\mathrm{Co} / \mathrm{Pt}$ multilayer, shows a stronger out-of-plane magnetic anisotropy on the $\mathrm{Bi}_{0.85} \mathrm{La}_{0.15} \mathrm{FeO}_{3}$ compared to the $\mathrm{BiFeO}_{3}$, Supplementary Fig. 13. Both these pieces of data provide substantiation that the out-of-plane magnetic exchange coupling is enhanced in the $\mathrm{Bi}_{0.85} \mathrm{La}_{0.15} \mathrm{FeO}_{3}$ sample; we attribute this enhancement to tilting of $\mathbf{M}_{\mathbf{C}}$ as a consequence of lanthanum substitution in $\mathrm{BiFeO}_{3}$.

Magnetoresistance measurements and micromagnetic simulations. Having established the changes in $\mathbf{P}, \mathbf{L}$, and $\mathbf{M}_{\mathbf{C}}$ as a function of lanthanum substitution as well as with electric field applied, captured schematically in Fig. 5a, we now proceed to ask the question: how do they impact the magnetotransport behavior of a spin-valve that is in magnetic contact with the $\mathrm{Bi}_{0.85} \mathrm{La}_{0.15^{-}}$ $\mathrm{FeO}_{3}$ surface. We deposited a spin-valve composed of $\mathrm{Pt}(2 \mathrm{~nm}) /$ $\mathrm{Co}_{0.9} \mathrm{Fe}_{0.1} \quad(2.5 \mathrm{~nm}) / \mathrm{Cu} \quad(5 \mathrm{~nm}) / \mathrm{Co}_{0.9} \mathrm{Fe}_{0.1} \quad(2.5 \mathrm{~nm})$ on the $\mathrm{Bi}_{0.85} \mathrm{La}_{0.15} \mathrm{FeO}_{3}$ surface and fabricated Hall-bar structures (Fig. 5b) to study the electrical-field dependence of magnetoresistance $[\mathrm{R}(\mathrm{H})]$ which is measured as the applied magnetic field, $H$, is swept from the positive value $H_{\max }$ to the negative value $-H_{\max }$ and back to zero field (sample preparation and device fabrication details are presented in the Methods section). We use the conventional form of magnetoresistance, defined as GMR (\%) $=\left[R(H)-R\left(H_{\max }\right)\right] \times 100 \% / R\left(H_{\max }\right)$. To draw the distinction, we compare a pure $\mathrm{BiFeO}_{3}$ layer to the $\mathrm{Bi}_{0.85} \mathrm{La}_{0.15} \mathrm{FeO}_{3}$ layer of the same thickness, Fig. $5 \mathrm{c}-\mathrm{f}$. We support the experimental data through micromagnetic simulations (details presented in Supplementary Information and Method section).

A typical GMR hysteresis loop as a function of electric bias (see the transport measurement details in Method Section) for the spin-valve on the pure $\mathrm{BiFeO}_{3}$ is shown in Fig. $5 \mathrm{c}$, with 
a
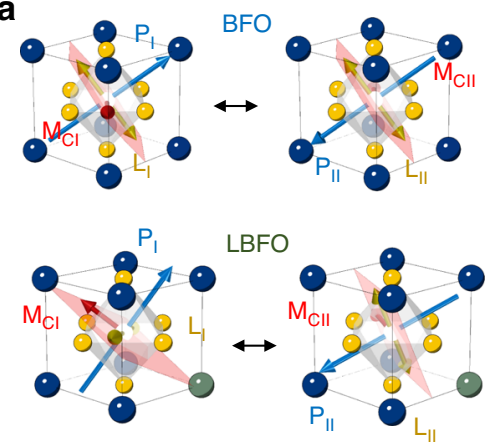

b

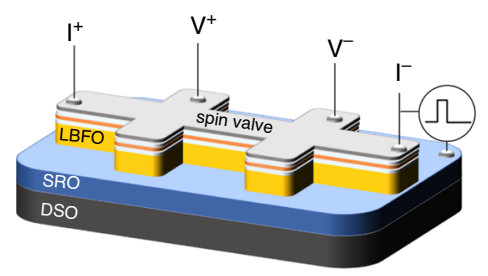

C

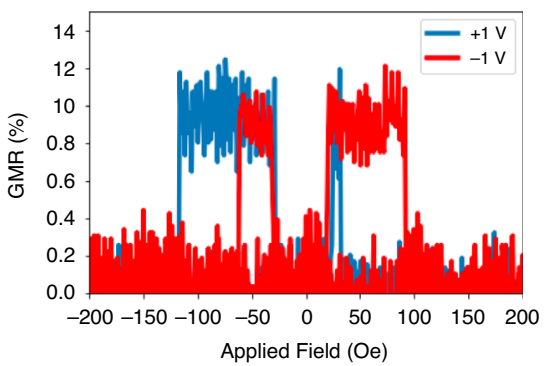

d

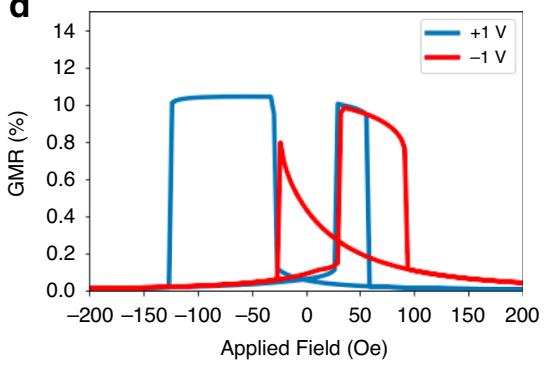

e

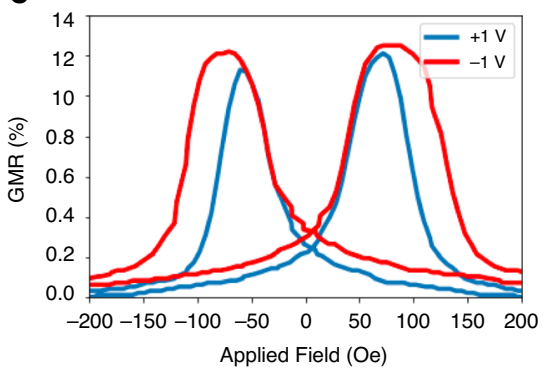

f

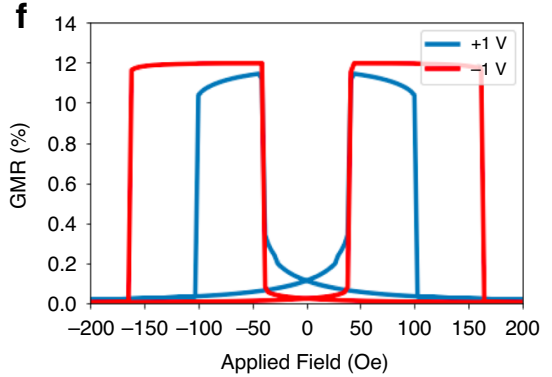

Fig. 5 Magnetic anisotropy switching by electric field via the heterostructure of spin-valve/ $\mathrm{BiFeO}_{\mathbf{3}}\left(\mathrm{Bi}_{\mathbf{0 . 8 5}} \mathrm{La}_{\mathbf{0 . 1 5}} \mathrm{FeO}_{\mathbf{3}}\right)$. a $\mathrm{Schematics}$ for the different electrically polarized states of $\mathbf{P}, \mathbf{L}$, and $\mathbf{M}_{\mathbf{C}}$ in $\mathrm{BiFeO}_{3}$ and $\mathrm{Bi}_{0.85} \mathrm{La}_{0.15} \mathrm{FeO}_{3}$. b Schematic for magnetoresistance measurements on spin-valve/BiFeO $\left(\mathrm{Bi}_{0.85} \mathrm{La}_{0.15} \mathrm{FeO}_{3}\right.$ ) heterostructure. $\mathbf{c} \mathrm{R}(\mathrm{H})$ of spin-valve/ $\mathrm{BiFeO}_{3}$ on the different electrically polarized states. d micromagnetic simulations on $\mathrm{R}(\mathrm{H})$ of spinvalve/ $\mathrm{BiFeO}_{3}$ with different polarization states. e $\mathrm{R}(\mathrm{H})$ of spin-valve/ $\mathrm{Bi}_{0.85} \mathrm{La}_{0.15} \mathrm{FeO}_{3}$ on the different electrically polarized states. $\mathbf{f}$ Micromagnetic simulations on $\mathrm{R}(\mathrm{H})$ of spin-valve/ $\mathrm{Bi}_{0.85} \mathrm{La}_{0.15} \mathrm{FeO}_{3}$ with different polarization states.

the corresponding micromagnetic simulation in Fig. 5d. Similarly, the GMR hysteresis loop for the spin-valve on $\mathrm{Bi}_{0.85} \mathrm{La}_{0.15} \mathrm{FeO}_{3}$ is shown in Fig. 5e, with the simulated data in Fig. 5f. For $\mathrm{BiFeO}_{3}$, we observe the modulation of an exchange bias by the applied electric field, which is consistent with our previous study ${ }^{34}$; on the other hand, for the $\mathrm{Bi}_{0.85} \mathrm{La}_{0.15} \mathrm{FeO}_{3}$ case, we observe the modulation of exchange coupling, leading to a reduction of the GMR switching field at positive electric field (additional details presented in Supplementary Fig. 14).

We can now put the data presented in Figs. 3-5 together to present the following observations. The insertion of lanthanum into $\mathrm{BiFeO}_{3}$ leads to a rotation of both $\mathbf{P}$ and $\mathbf{L}$ as well as their significantly different switching behaviors with an electric field. In the case of $\mathrm{Bi}_{0.85} \mathrm{La}_{0.15} \mathrm{FeO}_{3}$, the rotation of $\mathbf{M}_{\mathbf{C}}$ by $\sim 105^{\circ}$ amounts to an effective change in the magnetic anisotropy from essentially in-plane to essentially out-of-plane. This rotation of the magnetic anisotropy in $\mathrm{Bi}_{0.85} \mathrm{La}_{0.15} \mathrm{FeO}_{3}$ explains the E-field modulation of the exchange coupling between the $\mathrm{Co}_{0.9} \mathrm{Fe}_{0.1}$ and the $\mathrm{Bi}_{0.85} \mathrm{La}_{0.15^{-}}$ $\mathrm{FeO}_{3}$ layer that can be described by eq. (2). From Eq. (2), one can approximately derive the contributions to the energy of $\mathrm{Co}_{0.9} \mathrm{Fe}_{0.1}$ resulting from its interaction with $\mathrm{Bi}_{0.85} \mathrm{La}_{0.15} \mathrm{FeO}_{3}$ :

$$
F_{F M-A F M}=\mu_{0} H_{e b} M_{c}\left(\hat{m}_{c} \cdot \hat{m}\right)+K_{e c}(\hat{l} \cdot \hat{m})^{2}
$$

Here the lower-case symbols with a hat designate normalized vectors. Even though the values of exchange bias $\mathbf{H}_{\mathrm{eb}}$ and exchange coupling $K_{\mathrm{ec}}$ can be expressed via the exchange energy, their phenomenological values are more reliable, since they account for non-idealities of the interface. From the expression of exchange coupling energy, we can foresee that once we can control the anisotropy in the ferromagnet through the magnetoelectric coupling in $\mathrm{Bi}_{0.85} \mathrm{La}_{0.15} \mathrm{FeO}_{3}$, we will be able to switch its magnetization. This switch can be detected by measuring GMR in the spin-valve.
The micromagnetic simulations of the GMR stack (all the simulation details can be found in the Method and Supplementary Information) presented in Fig. $5 \mathrm{~d}$ and $\mathrm{f}$ show a close resemblance to the experimental data and provide theoretical credence to these experimental observations above. Compared to the parent phase $\mathrm{BiFeO}_{3}, \mathrm{Bi}_{0.85} \mathrm{La}_{0.15} \mathrm{FeO}_{3}$ shows a very different magnetoelectric switching where the magnetic anisotropy (mainly the magnetic easy plane) can be controlled by electric field while $\mathrm{BiFeO}_{3}$ has the same magnetic easy plane before and after the electric field is applied $25,32,34$.

\section{Discussion}

In summary, we have discovered that the polarization vector in the $\mathrm{BiFeO}_{3}$ system is systematically varied in both its magnitude and direction with respect to the crystal lattice, as the $\mathrm{Bi}^{+3}$ is replaced by $\mathrm{La}^{+3}$. The rotation of the polar vector away from the $\langle 111\rangle_{\mathrm{pc}}$ direction and towards the $\langle 112\rangle_{\mathrm{pc}}$ direction appears to be critical in terms of understanding both the anisotropy energy of the polar phase as well as the response of the system to out of plane electric fields. In addition, we demonstrate the ability for electrical control the anisotropy of magnetoelectric coupling through a spin-valve $/ \mathrm{Bi}_{0.85} \mathrm{La}_{0.15} \mathrm{FeO}_{3}$ device. Our results fulfill the understanding of magnetoelectric coupling in chemicallymodified $\mathrm{BiFeO}_{3}$ thin films, and can trigger the new application of multiferroics.

\section{Methods}

Film growth. The oxide heterostructures $\mathrm{Bi}_{0.85} \mathrm{La}_{0.15} \mathrm{FeO}_{3} / \mathrm{SrRuO}_{3}$ or $\mathrm{BiFeO}_{3} /$ $\mathrm{SrRuO}_{3}$ are grown on single-crystalline $(110)_{\mathrm{O}} \mathrm{DyScO}_{3}$ by pulse laser deposition at $690-710^{\circ} \mathrm{C}$ with focused laser fluence $\sim 1.2 \mathrm{~J} \mathrm{~cm}^{-2}$ under $100-160$ mTorr oxygen pressure and cooled down to room temperature at 500 Torr oxygen pressure. After the cooling process, the oxide heterostructures were immediately transferred to high vacuum magnetron sputtering chamber with a base pressure $\sim 1 \times 10^{-7}$ Torr. The spin-valve structures we used in this article, are fabricated with $\mathrm{Pt}(2.5 \mathrm{~nm}) /$ $\mathrm{Co}_{0.9} \mathrm{Fe}_{0.1}(2.5 \mathrm{~nm}) / \mathrm{Cu}(3-5 \mathrm{~nm}) / \mathrm{Co}_{0.9} \mathrm{Fe}_{0.1}(2.5 \mathrm{~nm})$, deposited by $\mathrm{DC}$ magnetron 
sputtering with argon pressure from ranging from $2 \times 10^{-3}$ to $7 \times 10^{-3}$ Torr under a static magnetic field of 200 Oe along the crystallographic [001 $]_{\mathrm{O}}$ to establish the magnetic easy axis. A $2 \mathrm{~nm}$ Pt layer is deposited on top of the spin-valve as a capping layer to protect the top $\mathrm{Co}_{0.9} \mathrm{Fe}_{0.1}$ layer from oxidation, whereas the bottom $\mathrm{SrRuO}_{3}$ layer serves as a bottom electrode for ferroelectric switching. The chemical composition analysis can be found in Supplementary Fig. 15.

\section{Scanning transmission electron microcopy and polarization mapping. TEM} samples were prepared by mechanical polishing with an Allied Multiprep followed by ion milling in a Gatan PIPS2. Samples were prepared so that the projected plane was $(1,0,0)$ c. HAADF-STEM images were collected on a JEOL JEM-ARM300CF operating at $300 \mathrm{kV}$.

In quantifying the polarization, we use the pseudocubic unit cell projected along the beam direction. The atomic polarization vector was calculated by measuring the displacement of $\mathrm{B} / \mathrm{A}$ site atoms from the center of the four surrounding $\mathrm{A} / \mathrm{B}$ site atoms in HAADF-STEM images. Each atom in the image is located accurately using gaussian fitting and the center of each unit cell is defined as the average position of the four atoms at the corners of the unit cell. Polarization mapping was performed on several images from different regions for the $\mathrm{BiFeO}_{3}$ and $\mathrm{Bi}_{0.85} \mathrm{La}_{0.15} \mathrm{FeO}_{3}$ samples. In bulk $\mathrm{BiFeO}_{3}$, the polarization in the $(100)_{\mathrm{pc}}$ projection plane should appear along the unit cell diagonal, $[001]_{\mathrm{pc}}$. To determine the deviation of the average polarization angle from the diagonal, we combined the data from several images together to generate one distribution for each sample. A Gaussian distribution was then fit to quantify the mean and standard deviation of the polarization angle in each sample.

\section{Fabrication of spin-valve devices and magnetoelectric coupling measure-} ments. Conventional photolithography and Ar-ion milling were employed to patterned the $\mathrm{Co}_{0.9} \mathrm{Fe}_{0.1}(2.5 \mathrm{~nm}) / \mathrm{Cu}(5 \mathrm{~nm}) / \mathrm{Co}_{0.9} \mathrm{Fe}_{0.1}(2.5 \mathrm{~nm})$ spin-valves devices of $15 \times 5 \mathrm{um}^{2}$. Subsequently, a $200 \mathrm{~nm}$ thick insulating amorphous $\mathrm{LaAlO}_{3}$ film was selectively deposited on $\mathrm{Bi}_{0.85} \mathrm{La}_{0.15} \mathrm{FeO}_{3}$ surface by PLD to isolate the connecting lines and contact pads from $\mathrm{Bi}_{0.85} \mathrm{La}_{0.15} \mathrm{FeO}_{3}$. DC magnetron sputtering was used to deposit $230 \mathrm{~nm}$ thick $\mathrm{Au}$ film for electric contacts. The GMR responses of spinvalve devices were measured in current-in-plane configuration at constant current with varying magnetic field. The magnetoelectric coupling measurements were conducted with the application of electric pulse $(10-100 \mu \mathrm{s})$ across $\mathrm{Bi}_{0.85} \mathrm{La}_{0.15} \mathrm{FeO}_{3}$ films.

Photoemission electron microscopy (PEEM). X-ray imaging with variable circular and linear polarization at the Co and Fe $L$ edges was performed at the PEEM3 end station of BL11.0.1 at the Advanced Light Source, Lawrence Berkeley National Lab. The sample was held at an angle of $60^{\circ}$ with respect to the surface normal, and was mounted on an azimuthal rotation stage. The in-plane azimuthal angle of zero degrees is with x-rays incident along the in-plane $[-100]_{\mathrm{pc}}$ direction $\left(\boldsymbol{\Phi}=0^{\circ}\right)$. The sample was held at a voltage of $-18 \mathrm{kV}$ to accelerate any photo-emitted and secondary electrons, proportional to the local $\mathrm{x}$-ray absorption coefficient, through a series of electrostatic lenses and incident onto a CCD detector with a phosphorcoated fiber plate serving as an amplifier. To probe antiferromagnetic orientation projections along the $\mathrm{x}$-ray linear polarization axis, linear dichroism images at the $\mathrm{Fe} L_{2} \mathrm{~A}$ and $\mathrm{B}$ edges of 720.6 and $722 \mathrm{eV}$ were taken at 5 linear polarization axis angles between s- and p-polarization (s: $\boldsymbol{\alpha}=0^{\circ} ; \mathrm{p}: \boldsymbol{\alpha}=90^{\circ}$ ). For each polarization value, a pre-edge image at $718 \mathrm{eV}$ was taken to normalize the on-edge images. A series of 10 images with $2 \mathrm{~s}$ acquisition time each were taken at each energy and polarization angle, and the entire polarization angle-energy sequence was repeated four times to improve statistics.

Piezoresponse force microscopy (PFM). PFM is performed with an Atomic Force Microscope (Asylum Research Cypher, Santa Barbara, Ca), conductive AFM probe (Nanoandmore, DT-NCHR, Watsonville, Ca) with DART mode. To determine domain orientation directions the normal $(\mathrm{z})$ and lateral ( $\mathrm{y}$ axis only) piezoresponse vectors are measured simultaneously by superimposing two small-signal $\mathrm{AC}$ biases with distinct frequencies corresponding to normal and lateral contact resonances of the AFM cantilever (approximately 300 and $600 \mathrm{kHz}$, respectively). The essentially binary phase signals are then vectorially combined to identify the four domain orientations present in the specimen.

Micromagnetic simulations. Micromagnetic simulations have been performed using the National Institute of Standards and Technology simulator, OOMMF. The inputs scripts for simulations are available in the Supplementary information. The simulation method follows closely to ref. ${ }^{34}$. Magnetization is calculated by minimizing the overall energy of the system at every value of the external magnetic field.

In contrast to $\mathrm{BiFeO}_{3}$ which exhibits periodic stripe domains, $\mathrm{Bi}_{0.85} \mathrm{La}_{0.15} \mathrm{FeO}_{3}$ has wide domains of single polarization. Thus, the antiferromagnetic order, $\mathbf{L}$ and canted magnetization, $\mathbf{M}_{\mathbf{C}}$, are assumed to be constant over the area of the device. The long axis of the measured spin-valve is along the $[100]_{p c}$ crystal axis. To make simulations run in an acceptable amount of computing time, the geometry of the $\mathrm{Co}_{09} \mathrm{Fe}_{01}$ layer was taken to be smaller than in experiments: $2000 \times 200 \times 1 \mathrm{~nm}^{3}$ with the in-plane grid of $20 \mathrm{~nm}$. This is justified since the contribution of the demagnetization (i.e. shape anisotropy) is small for this size range. To compensate for the ratio of the size to the exchange length in the ferromagnet, a lower value of the exchange stiffness was taken, $A_{x}=10 \mathrm{pJ} / \mathrm{m}$. Other parameters: $M_{s}=1 \mathrm{MA} / \mathrm{m}$. magnetization of $\mathrm{Co}_{0.9} \mathrm{Fe}_{0.1}, K_{e c}=5000 \mathrm{~J} / \mathrm{m}^{3}$ - in-plane anisotropy in the bottom layer due to the exchange coupling, exchange bias $H_{e b}=0 o e$. The normalized vectors mentioned above and used in simulations were at positive applied voltage $P_{\mathrm{I}}=\left[\begin{array}{ll}0.4082-0.4082 & 0.8165\end{array}\right] ; L_{\mathrm{I}}=\left[\begin{array}{l}0.82420 .5494-0.1374\end{array}\right] ; M_{\mathrm{CI}}=[-0.3925$

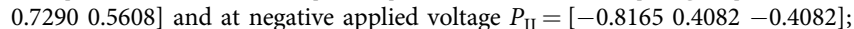
$L_{\mathrm{II}}=[0.44720-0.8944] ; M_{\mathrm{CII}}=[-0.3651-0.9129-0.1826]$. One can see that the projection of the canted magnetization on the shorter in-plane axis of the spinvalve is smaller than the long axis of the spin-valve. This explains why the manifestation of the exchange bias in the asymmetry of the hysteresis loop is smaller here than in ref. ${ }^{34}$.

\section{Data availability}

The data that support the findings of this study are available from the corresponding author upon reasonable request.

Received: 2 December 2019; Accepted: 21 May 2020;

Published online: 05 June 2020

\section{References}

1. Fiebig, M., Lottermoser, T., Meier, T. D. \& Trassin, M. The evolution of multiferroics. Nat. Rev. Mater. 1, 16046 (2016).

2. Spaldin, N. A. \& Fiebig, M. The renaissance of magnetoelectric multiferroics. Science 309, 391-392 (2005)

3. Chu, Y. H. et al. Electric-field control of local ferromagnetism using a magnetoelectric multiferroic. Nat. Mater. 7, 478-482 (2008).

4. Ramesh, R. \& Spaldin, N. A. Multiferroics: progress and prospects in thin films. Nat. Mater. 6, 21-29 (2007).

5. Spaldin, N. A. \& Ramesh, R. Advances in magnetoelectric multiferroics. Nat. Mater. 18, 203-212 (2019).

6. Manipatruni, S. et al. Scalable energy-efficient magnetoelectric spin-orbit logic. Nature 565, 35-42 (2019).

7. $\mathrm{Chu}, \mathrm{Y}$. H. et al. Low voltage performance of epitaxial $\mathrm{BiFeO}_{3}$ films on $\mathrm{Si}$ substrates through lanthanum substitution. Appl. Phys. Lett. 92, 102909 (2008).

8. Fusil, S., Garcia, V., Barthélémy, A. \& Bibes, M. Magnetoelectric devices for spintronics. Annu. Rev. Mater. Res. 44, 91-116 (2014).

9. Kiselev, S. V., Ozerov, R. P. \& Zhdanov, G. S. Detection of magnetic order in ferroelectric $\mathrm{BiFeO}_{3}$ by neutron diffraction. Sov. Phys. Dokl. 7, 742 (1963).

10. Filippetti, N. A. Why are there any magnetic ferroelectrics? J. Magn. Magn. Mater. 242, 976-979 (2002)

11. Hill, R. S. Visualizing the role of Bi $6 \mathrm{~s}$ "lone pairs" in the off-center distortion in ferromagnetic $\mathrm{BiMnO}_{3}$. Chem. Mater. 13, 2892-2899 (2001).

12. Saito, K. A. Structural characterization of $\mathrm{BiFeO}_{3}$ thin films by reciprocal space mapping. Jpn. J. Appl. Phys. 51, 7311 (2006).

13. Yang, C. H., Kan, D., Takeuchi, I., Nagarajan, V. \& Seidel, J. Doping $\mathrm{BiFeO}_{3}$ : approaches and enhanced functionality. Phys. Chem. Chem. Phys. 14, 15953-15962 (2012)

14. Kan, D. et al. Universal behavior and electric-field-induced structural transition in rare-earth-substituted $\mathrm{BiFeO}_{3}$. Adv. Funct. Mater. 20, 1108-1115 (2010).

15. Kan, D., Anbusathaiah, V. \& Takeuchi, I. Substitution-induced ferroelectric Polarization rotation in $\mathrm{BiFeO}_{3}$. Adv. Mater. 23, 1765-1769 (2011).

16. Karpinsky, D. V. et al. Evolution of crystal structure and ferroic properties of La-doped $\mathrm{BiFeO}_{3}$ ceramics near the rhombohedral-orthorhombic phase boundary. J. Alloy Compd. 555, 101-107 (2013).

17. Jang, B. K. et al. Electric-field-induced spin disorder-to-order transition near a multiferroic triple phase point. Nat. Phys. 13, 189-196 (2017).

18. Jang, H. W. et al. Strain-induced polarization rotation in epitaxial (001) $\mathrm{BiFeO}_{3}$ thin films. Phys. Rev. Lett. 101, 107602 (2008).

19. Kiat, J. M. et al. Monoclinic structure of unpoled morphotropic high piezoelectric PMN-PT and PZN-PT compounds. Phys. Rev. B 65, 064106 (2002).

20. Heron, J. T. et al. Deterministic switching of ferromagnetism at room temperature using an electric field. Nature 516, 370-373 (2014).

21. Dzialoshinskii, I. E. Thermodynamic theory of weak ferromagnetism in antiferromagnetic substances. Sov. Phys. JETP 5, 1259 (1957).

22. Ederer, C. \& Spaldin, N. A. Weak ferromagnetism and magnetoelectric coupling in bismuth ferrite. Phys. Rev. B 71, 060401 (2005).

23. Sosnowska, I., Neumaier, T. P. \& Steichele, E. Spiral magnetic ordering in bismuth ferrite. J. Phys. C Solid State Phys. 15, 4835 (1982)

24. Feiming, B. et al. Destruction of spin cycloid in $(111)_{C}$-oriented $\mathrm{BiFeO}_{3}$ thin films by epitiaxial constraint: enhanced polarization and release of latent magnetization. Appl. Phys. Lett. 86, 032511 (2005). 
25. Zhao, T. et al. Electrical control of antiferromagnetic domains in multiferroic $\mathrm{BiFeO}_{3}$ films at room temperature. Nat. Mater. 5, 823-829 (2006).

26. Béa, H., Bibes, M., Petit, S., Kreisel, J. \& Barthélémy, A. Structural distortion and magnetism of $\mathrm{BiFeO}_{3}$ epitaxial thin films: a Raman spectroscopy and neutron diffraction study. Philos. Mag. Lett. 87, 165 (2007).

27. Chen, Z. et al. Complex strain evolution of polar and magnetic order in multiferroic $\mathrm{BiFeO}_{3}$ thin films. Nat. Commun. 9, 3764 (2018).

28. $\mathrm{Chu}, \mathrm{Y}$. H. et al. Nanoscale control of domain architectures in $\mathrm{BiFeO}_{3}$ thin films. Nano Lett. 9, 1726-1730 (2009).

29. Nolting, F. et al. Direct observation of the alignment of ferromagnetic spins by antiferromagnetic spins. Nature 405, 767-769 (2000)

30. Scholl, A. et al. Observation of antiferromagnetic domains in epitaxial thin films. Science 287, 1014-1016 (2000).

31. Ohldag, $\mathrm{H}$. et al. Spin reorientation at the antiferromagnetic $\mathrm{NiO}(001)$ surface in response to an adjacent ferromagnet. Phys. Rev. Lett. 86, 2878 (2001).

32. Holcomb, M. B. et al. Probing the evolution of antiferromagnetism in multiferroics. Phys. Rev. B 81, 134406 (2010).

33. Kuo, C.-Y. et al. Single-domain multiferroic $\mathrm{BiFeO}_{3}$ films. Nat. Commun. 7, $12712(2016)$

34. Manipatruni, S. et al. Voltage control of unidirectional anisotropy in ferromagnet-multiferroic system. Sci. Adv. 4, eaat4229 (2018).

\section{Acknowledgements}

Y.-L.H. was supported by the LBNL LDRD project on Beyond Moore's Law Electronics. L.W.M. and R.R. acknowledge support of Intel Corp. through the FEINMAN program. M. Y. and Z. Q. Qiu acknowledge support by US Department of Energy, Office of Science, Office of Basic Energy Sciences, Materials Sciences and Engineering Division under Contract No. DE-AC02-05CH11231 (van der Waals heterostructures program, KCWF16). This research used resources of the Advanced Light Source, which is a DOE Office of Science User Facility under contract no. DE-AC02-05CH11231.

\section{Author contributions}

Y.-L.H. and R.R. designed the experiments and wrote this paper. Y.-L.H. carried out materials synthesis and characterization. D.V. conducted the micromagnet simulations on the magnetoelectric measurements. C.A. carried the STEM measurements, analysis, and supervised by X. P. Y.-L.H and B.P performed device fabrication and electrical measurements. Y.-L.H., L.Z., H.-J.L., and Y.-H.C. performed the XRD measurements. Y.-L.H., M.R., and B.D.H. conducted the PFM measurements and analysis. J.C., M.Y., Z.Q.Q. and J.B. performed the magneto-optic measurements. Additional device processing, electrical measurements, and analysis were carried out by T.G., J.C., C.-C.L., and I.Y. R.V.C, Y.-L.H., and A.F. performed the XMLD-PEEM measurements and R.V.C provided analysis. J.I. performed the calculations for the ferroelectric switching energy. All authors made contribution to writing the manuscript. The work was conceived and guided by R.R. and L.W.M. All authors discussed results and commented on the manuscript.

\section{Competing interests}

The authors declare no competing interests.

\section{Additional information}

Supplementary information is available for this paper at https://doi.org/10.1038/s41467020-16727-2.

Correspondence and requests for materials should be addressed to R.R.

Peer review information Nature Communications thanks the anonymous reviewer(s) for their contribution to the peer review of this work.

Reprints and permission information is available at http://www.nature.com/reprints

Publisher's note Springer Nature remains neutral with regard to jurisdictional claims in published maps and institutional affiliations.

(c) (i) Open Access This article is licensed under a Creative Commons Attribution 4.0 International License, which permits use, sharing, adaptation, distribution and reproduction in any medium or format, as long as you give appropriate credit to the original author(s) and the source, provide a link to the Creative Commons license, and indicate if changes were made. The images or other third party material in this article are included in the article's Creative Commons license, unless indicated otherwise in a credit line to the material. If material is not included in the article's Creative Commons license and your intended use is not permitted by statutory regulation or exceeds the permitted use, you will need to obtain permission directly from the copyright holder. To view a copy of this license, visit http://creativecommons.org/ licenses/by/4.0/.

(C) The Author(s) 2020 